\title{
I.V.Bagrova, G.A.Kukharchik, V.I.Serebryakova \\ Prognostic factors of poor outcome in patients with ischemic heart disease and pulmonary embolism
}

\begin{abstract}
Summary
Patients with ischemic heart disease (IHD) have a high risk of the death during the first year after an episode of pulmonary embolism (PE). This study involved 71 patients with IHD and PE. Data of medical history, clinical manifestations and laboratory findings were analyzed. Factors predictive for poor outcome in patients with IHD and PE were age $>65 \mathrm{yrs,} \mathrm{concomitant} \mathrm{chronic} \mathrm{obstructive} \mathrm{pulmonary} \mathrm{disease,} \mathrm{malignancies,} \mathrm{seizures}$ and severe hypertension as clinical manifestations of $\mathrm{PE}$, the left ventricle ejection fraction $<45 \%$, pulmonary artery systolic pressure $>50 \mathrm{mmHg}$, diameter of the pulmonary artery $>28 \mathrm{~mm}$.

Key words: pulmonary embolism, ischemic heart disease, prognosis, mortality.
\end{abstract}

Резюме

У больных ишемической болезнью сердца (ИБС) после эпизода тромбоэмболии легочной артерии (ТЭЛА) сохраняется высокий риск летального исхода в течение одного года. В исследование был включен 71 пациент с ИБС в сочетании с ТЭЛА. Были проанализированы анамнестические данные, клинические проявления, данные инструментального исследования у этих пациентов. Выявлены следующие предикторы неблагоприятного исхода при ТЭЛА у больных ИБС: возраст $>65$ лет, сопутствующие хроническая обструктивная болезнь легких и новообразования, клиническая манифестация ТЭЛА в виде синкопального состояния и тяжелой артериальной гипотензии, фракция левого желудочка $<45 \%$, систолическое давление в легочной артерии $>50$ мм рт. ст. и диаметр легочной артерии $>28$ мм. Ключевые слова: тромбоэмболия легочной артерии, ишемическая болезнь сердца, прогноз, летальность

Актуальность проблемы тромбоэмболии легочной артерии (ТЭЛА) связана с ее большой распространенностью и частой сочетаемостью с различными заболеваниями, которые могут оказывать влияние на проявления и прогноз ТЭЛА, а также с высокой летальностью при этой патологии [1-3]. По данным эпидемиологических исследований, частота ТЭЛА в популяции составляет от 0,50 до 2,08 на 1000 населения в год, а у лиц старше 75 лет этот показатель достигает 1 на 100 в год [1, 3, 4]. По мнению разных исследователей, смертность при ТЭЛА варьируется в широких пределах - от 1 до $30 \%$ [2, 5-7]. Согласно результатам многоцентровых исследований по изучению легочной эмболии, риск летального исхода после перенесенной ТЭЛА сохраняется в течение нескольких месяцев, особенно на протяжении 1-го мес. после эпизода тромбоэмболии [4, 7, 8]. Среди факторов неблагоприятного исхода в литературе отмечают мужской пол [4], возраст старше 75 лет [6], длительную иммобилизацию [4, 6], клиническую манифестацию ТЭЛА в виде артериальной гипотензии $[9,10]$, тахикардии $[9,11]$, проявления дисфункции правого желудочка (ПЖ) [10, 12], положительный тест на тропонин-Т или -I [3], из сопутствующих заболеваний - новообразования $[4,10]$, хронические заболевания легких [10].
В структуре сопутствующей ТЭЛА патологии 1-е место занимают сердечно-сосудистые заболевания [2]. По мнению В.Г.Мишалова, среди причин смертности от сердечно-сосудистых заболеваний на долю ТЭЛА приходятся 50-60\% [13]. Представляется актуальным выявление прогностических факторов неблагоприятного исхода при сочетании ТЭЛА с ишемической болезнью сердца (ИБС) для стратификации риска и определения тактики ведения больного, в т. ч. своевременного проведения тромболизиса или выполнения эмболэктомии.

Целью настоящего исследования явилась оценка прогностической значимости факторов риска неблагоприятного исхода при ТЭЛА у больных ИБС.

\section{Материалы и методы}

Был обследован 71 больной с ТЭЛА в сочетании с ИБС. Все пациенты находились на стационарном лечении в одной из городских больниц Санкт-Петербурга в период 2005-2009 гг. Диагноз ТЭЛА основывался на анализе клинической картины (появление одышки или удушья, болей в грудной клетке, тахикардии, артериальной гипотензии, потери сознания), данных инструментального исследования (электрокардиографии, эхокардиографии, рентгено- 
графии органов грудной клетки, перфузионной сцинтиграфии легких, компьютерной томографии органов грудной клетки, ангиопульмонографии), позволивших подтвердить диагноз ТЭЛА. Все больные получали стандартную терапию.

Наблюдение за пациентами осуществлялось в течение 1 года. За этот период умерли 35 человек. Для определения значимости предикторов неблагоприятного исхода ТЭЛА все больные были разделены на 2 группы в зависимости от исхода. В 1-ю группу вошли 35 пациентов, умерших в течение года, во 2-ю - 36 выживших больных. Среди пациентов 1-й группы 7 человек умерли в 1-е сут. пребывания в стационаре, поэтому они были исключены из исследования. Таким образом, поиск предикторов неблагоприятного исхода проводился среди 28 больных с неблагоприятным исходом и 36 больных с благоприятным исходом.

Статистическая обработка материала проводилась посредством программы Excel, а статистические расчеты - с использованием программы Statistica 8.0. Достоверность различий средних значений показателей определяли с помощью t-критерия Стьюдента. Для проверки гипотезы о равенстве дисперсий выборок пользовались критерием Фишера. Если вычисленное значение этого критерия превышало соответствующее табличное значение, гипотеза о равенстве дисперсий отвергалась и разница средних арифметических оценивалась по формуле с числом степеней свободы. Достоверность различий качественных признаков в сравниваемых группах оценивали при помощи критерия $\chi^{2}$ и точного метода Фишера. Для выявления предикторов неблагоприятного исхода при ТЭЛА в сочетании с ИБС использовали модель пропорциональных рисков Кокса.

\section{Результаты}

Под наблюдением в стационаре находился 71 больной. Среди пациентов было 43 женщины $(60,6 \%)$ и 28 мужчин (39,4\%), средний возраст - 66,2 \pm 8,9

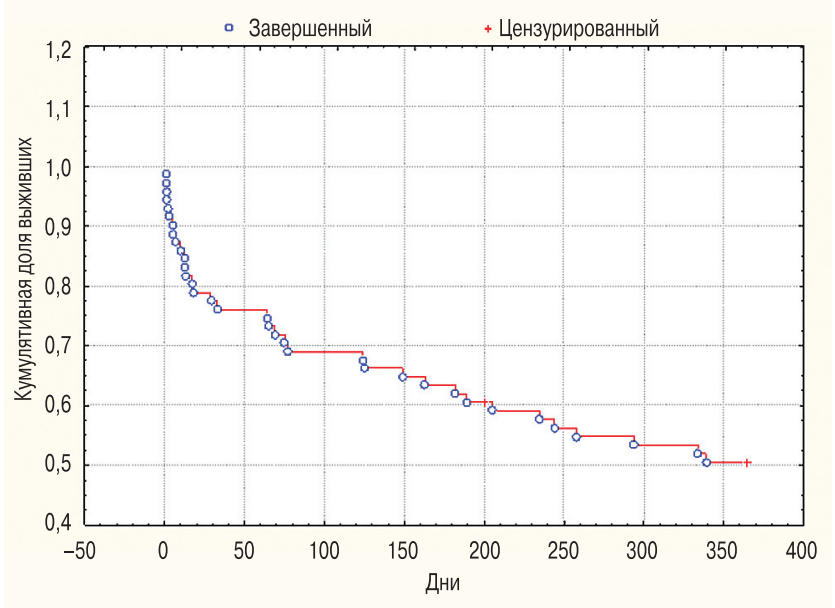

Рисунок. Кривая выживаемости (Каплан-Мейера) пациентов с ТЭЛА в сочетании с ИБС года. Уровень летальности пациентов с ТЭЛА при ИБС в течение 1 года составил 49,3\% (рисунок). Максимальное количество летальных исходов регистрировалось в течение 1-го месяца и достигло 17 случаев (23,9\%), в течение 3 мес. умерли 23 пациента $(32,4 \%)$.

При анализе распределения больных по полу значимых отличий не получено. Так, пациенты в 1-й группы были старше, чем во 2-й, в среднем на 5 лет $(p<0,05)$. В 1-й группе средний возраст больных составил $68,4 \pm 8,0$ года, а во $2-и ̆ ~-63,1 \pm 12,1$ года. При этом в 1-й группе был 21 пациент $(75,0$ \%) 65 лет и старше, а во 2-й - 16 человек того же возраста $(44,4 \% ; p<0,05)$.

ИБС была представлена постинфарктным кардиосклерозом и стенокардией напряжения различного функционального класса. Группы значимо не различались по клиническим формам ИБС. Проявления хронической сердечной недостаточности $(\mathrm{XCH})$ были выявлены у всех 64 пациентов, при анализе распространенности по степени тяжести ХСН между группами значимых отличий не получили, но в обеих группах преобладала XCH III-IV функционального класса - у 22 больных $(78,6 \%)$ в 1-й группе и у 26 пациентов $(72,2 \%)$ - во 2-й.

В группе больных с неблагоприятным исходом чаще выявлялись хроническая обструктивная болезнь легких (ХОБЛ), новообразования. Так, ХОБЛ была диагностирована у 7 пациентов $(25,0 \%)$ 1-й группы и у 2 больных $(5,6 \%)$ 2-й группы. Новообразования были обнаружены у 4 человек (14,3\%) в 1-й группе, у больных 2-й группы они не выявлены.

Манифестация ТЭЛА у пациентов с неблагоприятным исходом была чаще связана с потерей сознания и артериальной гипотензией. Синкопальное состояние развилось у 9 пациентов $(32,1 \%) 1$-й группы и 3 больных $(8,3 \%)-2$-й $(p<0,05)$. Снижение систолического артериального давления < 90 мм рт. ст. зарегистрировано у 8 больных $(28,6 \%) 1$-й группы и 3 пациентов $(8,3 \%)-2$-й $(p<0,05)$.

При исследовании параметров гемодинамики выявлено, что фракция выброса левого желудочка (ФВ ЛЖ) составила 44,0 $\pm 13,9 \%$ у пациентов 1 -й группы и 54,0 \pm 7,2\% у пациентов 2-й группы $(p<0,05)$, т. е. в случае летального исхода она была в среднем на $10 \%$ ниже. При этом снижение ФВ ЛЖ до $\leq 45 \%$ зарегистрировано у 6 пациентов $(28,6 \%)$ с летальным исходом и 1 больного $(4,3 \%)$ с благоприятным исходом. Диаметр легочной артерии (ЛА) в 1-й группе в среднем составил $26,4 \pm 3,2$ мм, а во 2 -й - 24,9 $\pm 1,7$ мм $(p<0,05)$. Систолическое давление в легочной артерии (СДЛА) было в среднем на 18 мм рт. ст. больше у пациентов с летальным исходом и составило у больных 1-й группы 68,0 \pm 15,3 мм рт. ст., а во 2-й группе $-49,5 \pm 12,6$ мм рт. ст. $(p<0,05)$. Повышение СДЛА > 50 мм рт. ст. выявлено у 47,8 \% больных с благоприятным исходом и у $80,9 \%$ - с летальным $(p<0,05)$.

Чтобы изучить влияние сочетания факторов на развитие неблагоприятного исхода, был использован метод пропорциональных интенсивностей 
Кокса. Полученная модель имеет статистическую оценку $\chi^{2}-24,7$ ( $\left.p=0,002\right)$, как показано в таблице.

Пациентам с благоприятным исходом выполнили повторное эхокардиографическое исследование через 1 год. Значение ФВ ЛЖ в динамике не изменилось, составив 54,0 \pm 7,2 \% исходно и 55,0 \pm 8,5 \% через год $(p>0,05)$. Направленность изменений СДЛА при сравнении в динамике была различной. В целом отмечено значимое уменьшение СДЛА с 49,5 \pm 12,6 до $35,2 \pm 12,1$ мм рт. ст., в среднем на 14 мм рт. ст. $(p<0,05)$. При этом у 16 пациентов $(69,7 \%)$ СДЛА снизилось до $\leq 30$ мм рт. ст., а у 5 человек $(21,6 \%)$ оставалось высоким или даже продолжало нарастать. У 2 больных (8,7 \%) отмечено увеличение СДЛА в течение года до 50-80 мм рт. ст.

\section{Обсуждение}

В проведенном исследовании был подтвержден высокий риск неблагоприятного исхода у пациентов с ТЭЛА в сочетании с ИБС. По данным литературы, максимальная летальность при ТЭЛА регистрируется в течение 1-го мес. [7, 9]. В нашем исследовании смертность за 1-й мес. также была наиболее высокой и составила 23,9\%. Летальность за 3 мес. достигла $32,4 \%$. Этот показатель больше, чем в регистре ICOPER (17,5\%) [7] и регистре RIETE $(8,7 \%)$ [6].

Известно, что с возрастом риск ТЭЛА повышается. По данным многоцентрового регистра RIETE, включившего 15520 пациентов с тромбозом глубоких вен и / или ТЭЛА, прогностически неблагоприятным являлся возраст > 75 лет [6]. В настоящем исследовании риск летального исхода был выше у пациентов старше 65 лет.

Прогностически неблагоприятными в структуре сопутствующих заболеваний у наблюдаемых пациентов были ХОБЛ и новообразования. Полученные результаты совпадают с данными N.Kucher et al., выявившими, что наличие заболеваний легких у пациентов с ТЭЛА являлось предиктором неблагоприятного исхода в течение 30 дней [7]. Роль новообразований в развитии летального исхода также подтверждена в исследованиях S.Laporte et al. и M.Nacamura et al. [4, 6].
Полученные нами данные о важности артериальной гипотензии (как клинической манифестации ТЭЛА) в развитии летального исхода сопоставимы с имеющимися литературными данными. На значимость артериальной гипотензии указывали D.Aujesky et al., N.Kucher et al., J.L.Lobo et al. [8-11]. Потеря сознания также отмечается разными исследователями как одно из клинических проявлений ТЭЛА $[9,10]$. Прогностически неблагоприятная роль тахикардии, установленная D.Aujesky et al. [9], в настоящем исследовании не подтвердилась. Учащение частоты сердечных сокращений отмечалось у больных ТЭЛА в сочетании с ИБС независимо от клинического исхода.

По данным регрессионного анализа Кокса (среди всех включенных в анализ факторов), ФВ ЛЖ < $45 \%$ оказалась в большей степени связанной с риском развития неблагоприятного исхода при ТЭЛА в сочетании с ИБС (коэффициент beta - 0,91; ОШ 9,86; 95\%-ный ДИ - 1,07-90,91; $p<0,05)$.

К числу предикторов неблагоприятного исхода при ТЭЛА относят дисфункцию ПЖ как проявление острого легочного сердца $[10,12]$. По данным S.Grifoni et al., признаком острой дисфункции ПЖ является наличие по крайней мере одного из признаков: дилатация ПЖ (конечный диастолический размер - > 30 мм), парадоксального систолического движения межжелудочковой перегородки, легочной гипертензии с СДЛА > 30 мм рт. ст. [12].

В настоящем исследовании частота выявления признаков дисфункции ПЖ у пациентов с ТЭЛА на фоне ИБС составила 95,8 \% у больных в 1-й группы и 100,0 \% - во 2-й. Эти данные выше показателей, полученных S.Grifoni et al. (48,5 \%) [12] и N.Kucher et al. (39\%) [10]. Признаки дисфункции ПЖ у пациентов с ТЭЛА в сочетании с острым инфарктом миокарда, по данным И.В.Прохоровой, были выявлены у 64,7 \% больных [14]. Поскольку, независимо от исхода, признаки дисфункции ПЖ наблюдались у большинства пациентов с ТЭЛА в сочетании с ИБС, были детально проанализированы отдельные показатели гемодинамики. Оказалось, что прогностически неблагоприятными являются повышение СДЛА $>50$ мм рт. ст. и увеличение диаметра ЛА > 28 мм.

Отношение шансов (ОШ) неблагоприятного исхода у пациентов при ТЭЛА в сочетании с ИБС для наиболее информативных показателей в модели Кокса

\begin{tabular}{|c|c|c|c|c|c|}
\hline \multirow[t]{2}{*}{ Признак } & \multirow[t]{2}{*}{ Beta } & \multirow[t]{2}{*}{ ОШ } & \multicolumn{2}{|c|}{ 95\%-ный ДИ } & \multirow[t]{2}{*}{$p$} \\
\hline & & & нижняя граница & верхняя граница & \\
\hline Возраст > 65 лет & 0,69 & 3,75 & 1,28 & 11,05 & $<0,05$ \\
\hline ХОБЛ & 0,36 & 5,67 & 1,07 & 29,9 & $<0,05$ \\
\hline Новообразования & 1,01 & - & - & - & $<0,05$ \\
\hline Потеря сознания & 0,97 & 5,2 & 1,26 & 21,67 & $<0,05$ \\
\hline Артериальная гипотензия & 0,24 & 4,4 & 1,04 & 18,58 & $<0,05$ \\
\hline ФВ ЛЖ < 45 \% & 0,91 & 9,86 & 1,07 & 90,91 & $<0,05$ \\
\hline СДЛА > 50 мм рт. ст. & 0,51 & 6,70 & 1,55 & 29,09 & $<0,05$ \\
\hline Диаметр ЛА > 28 мм & 0,77 & 7,33 & 1,34 & 40,30 & $<0,05$ \\
\hline
\end{tabular}

Примечание: 95\%-ный ДИ - 95\%-ный доверительный интервал. 


\section{Заключение}

Проведенное исследование показало высокий риск летального исхода при ТЭЛА у больных ИБС в течение 1 года. Были выявлены следующие предикторы неблагоприятного исхода: возраст > 65 лет, манифестация ТЭЛА в виде потери сознания и артериальной гипотензии, сопутствующая ХОБЛ и злокачественные новообразования, снижение ФВ ЛЖ $<45 \%$, повышение СДЛА > 50 мм рт. ст., увеличение диаметра ЛА > 28 мм.

\section{Литература}

1. Бунин Ю.А. Диагностика и лечение тромбоэмболии легочной артерии: современное состояние проблемы. Трудный пациент 2005; 4: 26-33.

2. Крахмалова Е.О. Хроническая постэмболическая легочная гипертензия: терапевтические аспекты. Серцева недостатність 2010; 1: 45-61.

3. Torbicki A., Perrier A., Konstantinides S. et al. Guidelines on the diagnosis and management of acute pulmonary embolism: The task force for the diagnosis and management of acute pulmonary embolism of the European Society of Cardiology (ESC). Eur. Heart J. 2008; 29 (18): 2276-2315.

4. Nakamura M., Fujioka H., Yamada N. et al. Clinical characteristics of acute pulmonary thromboembolism in Japan: Results of a multiccnter registry in the Japanese Society of Pulmonary Embolism Research. Clin. Cardiol. 2001; 24: 132-138.

5. Никитин А.В. Роль неинвазивной диагностики тромбоэмболии легочной артерии в оптимизации лечения: Автореф. дис. ... канд. мед. наук. СПб.; 2001.

6. Laporte S., Mismetti P., Decousus H. et al. Clinical predictors for fatal pulmonary embolism in 15520 patients with venous thromboembolism. Findings from the Registro Informatizado de la Enfermedad TromboEmbolica venosa (RIETE) registry. Circulation 2008; 117: 1711-1716.

7. Kucher N., Rossi E., De Rosa M., Goldhaber S.Z. Massive pulmonary embolism. Circulation 2006; 113: 577-582.
8. Lobo J.L., Zorrilla V., Aizpuru F. et al. Clinical syndromes and clinical outcome in patients with pulmonary embolism. Findings from the RIETE registry. Chest 2006; 130: 1817-1822.

9. Aujesky D., Obrosky D. S., Stone R.A. et al. A prediction rule to identify low-risk patients with pulmonary embolism. Arch. Intern. Med. 2006; 166: 169-175.

10. Kucher N., Rossi E., De Rosa M. et al. Prognostic role of echocardiography among patients with acute pulmonary embolism and a systolic arterial pressure of $90 \mathrm{~mm} \mathrm{Hg}$ or higher. Arch. Intern. Med. 2005; 165: 1777-1781.

11. Aujesky D., Roy P.M., Le Manach C.P. et al. Validation of a model to predict adverse outcomes in patients with pulmonary embolism. Eur. Heart J. 2006; 27: 476-481.

12. Grifoni S., Vanni S., Magazzini S. et al. Association of persistent right ventricular dysfunction at hospital discharge after acute pulmonary embolism with recurrent thromboembolic events. Arch. Intern. Med. 2006; 166: 2151-2156.

13. Мишалов В.Г., Амосова Е.Н., Мостовой Ю.М. Тромбоэмболия легочной артерии: дискуссия продолжается. Здоров`я Украіни 2007; 5: 5-7.

14. Прохорова И.В. Частота и особенности диагностики тромбоэмболии легочной артерии при остром инфаркте миокарда: Автореф. дис. ... канд. мед. наук. Томск; 2006.

\section{Информация об авторах}

Багрова Ирина Викторовна - ассистент кафедры госпитальной терапии с курсами семейной медицины, клинической фармакологии и клинической лабораторной диагностики СПбГМА им. И.И.Мечникова тел.: (812) 545-37-77; e-mail: irinbagrov@yandex.ru

Кухарчик Галина Александровна - к. м. н., доцент, очный докторант кафедры госпитальной терапии с курсами семейной медицины, клинической фармакологии и клинической лабораторной диагностики СПбГМА им. И.И.Мечникова; тел.: (812) 545-37-77; e-mail: gkukharchik @yandex.ru

Серебрякова Валентина Ивановна - проф. кафедры госпитальной терапии с курсами семейной медицины, клинической фармакологии и клинической лабораторной диагностики СПбГМА им. И.И.Мечникова; тел.: (812) 545-37-77; e-mail: 